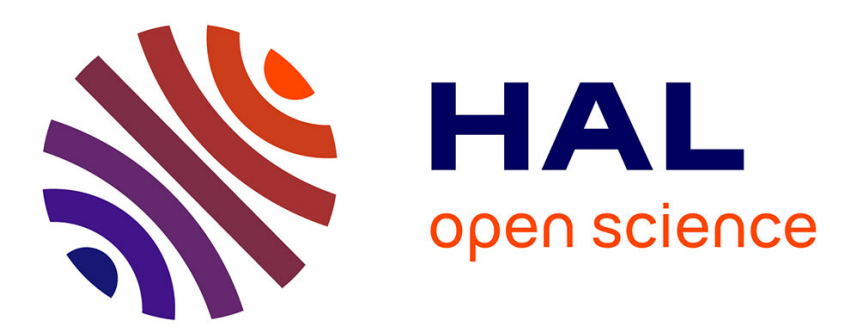

\title{
High-resolution electrical characterization of RuO2-borosilicate glass composites
}

Andrea Piarristeguy, Rafael Nuernberg, Dylan Jouglard, Michel Ramonda, Richard Arinero, Annie Pradel, Muriel Neyret

\section{To cite this version:}

Andrea Piarristeguy, Rafael Nuernberg, Dylan Jouglard, Michel Ramonda, Richard Arinero, et al.. High-resolution electrical characterization of $\mathrm{RuO} 2$-borosilicate glass composites. Journal of Alloys and Compounds, 2021, 876, pp.160123. 10.1016/j.jallcom.2021.160123 . hal-03225727

\section{HAL Id: hal-03225727 \\ https://hal.science/hal-03225727}

Submitted on 12 May 2021

HAL is a multi-disciplinary open access archive for the deposit and dissemination of scientific research documents, whether they are published or not. The documents may come from teaching and research institutions in France or abroad, or from public or private research centers.
L'archive ouverte pluridisciplinaire HAL, est destinée au dépôt et à la diffusion de documents scientifiques de niveau recherche, publiés ou non, émanant des établissements d'enseignement et de recherche français ou étrangers, des laboratoires publics ou privés. 


\title{
High-resolution electrical characterization of $\mathrm{RuO}_{2}$-borosilicate glass composites
}

\author{
Andrea Piarristeguy ${ }^{\mathrm{a}}$, Rafael Nuernberg ${ }^{\mathrm{b}}$, Dylan Jouglard ${ }^{\mathrm{b}}$, Michel Ramonda ${ }^{\mathrm{c}}$, Richard Arinero ${ }^{\mathrm{d}}$, \\ Annie Pradel ${ }^{\mathrm{a}}$, Muriel Neyret ${ }^{\mathrm{b}}$ \\ a ICGM, Univ Montpellier, CNRS, ENSCM, Montpellier, France \\ b CEA, DEN, DE2D/SEVT/LDMC - Marcoule, F-30207 Bagnols-sur-Cèze, France \\ c Centre de Technologie de Montpellier, Université de Montpellier, France \\ ${ }^{\mathrm{d}}$ Institut d'Électronique et des Systèmes, Université de Montpellier, Montpellier, France
}

\section{A R T I C L E I N F O}

\section{Article history:}

Received 3 February 2021

Received in revised form 12 April 2021

Accepted 22 April 2021

Available online xxx

\section{Keywords}

Nuclear waste management

Nuclear glasses

Ruthenium oxide

Electrical conductivity

Dielectric properties

Near field microscopy

\begin{abstract}
A B S T R A C T
$\mathrm{RuO}_{2}$-borosilicate glass composites representing nuclear glasses show a sudden increase in electrical conductivity when the $\mathrm{RuO}_{2}$ content reached a concentration of around $1 \%$ in volume. This percolation threshold is astonishingly low compared to a geometrical percolation threshold, i.e. 15\%, considering the random distribution of phases. Herein, electrostatic force microscopy (EFM) analyses were used to investigate this anomalous behavior by performing local dielectric characterization of the $\mathrm{RuO}_{2}$-borosilicate glass composites that comprised clusters of precipitated $\mathrm{RuO}_{2}$ particles and "apparently" $\mathrm{RuO}_{2}$-free borosilicate regions. As expected, differences between the relative permittivity of the two zones, borosilicate matrix, and $\mathrm{RuO}_{2}$ particles, were found. Most interesting, the sensitivity of the EFM technique combined with numerical modeling based on a finite element method revealed that the relative permittivity of the borosilicate matrix was enhanced with the overall $\mathrm{RuO}_{2}$ content. Such an increase can be explained by a local enrichment of the borosilicate matrix in $\mathrm{RuO}_{2}$, which increases the overall relative permittivity due to its much higher electronic polarizability. The enrichment of the matrix could play a role in the conduction mechanism, changing the effective volume of the conductive medium and, therefore, decreasing the minimal volume of the $\mathrm{RuO}_{2}$ phase necessary for the electrical percolation.
\end{abstract}

(C) 2021

\section{Introduction}

Nowadays, the internationally recognized standard treatment for high-level nuclear waste is immobilization through vitrification, followed by storage. Substantial improvements have been implemented on induction melters such as the cold crucible induction melter (CCIM). The most important breakthrough linked to the CCIM technology arises from the protective layer of cooled glass, called the "self-crucible," which forms on the crucible inner wall. This glass is both thermally insulating and anticorrosive, which allows high-temperature operation and corrosive melts. The CCIM uses radiofrequency induction to power the glass melt itself rather than the lower frequency induction of the metal shell employed in conventional melters. This process is characterized by directly induced currents inside the molten glass through the penetration of the electromagnetic field into its volume enabled by a coil. The absorption of electromagnetic radiation allows the melt to be directly heated by Joule effect without heating the crucible. Besides, the crucible walls are cooled by a water circulation system making the layer of cooled glass transparent to the electromagnetic field. The electrical properties of the glass and the melt, such as conductivity, permittivity, and loss, are of primary interest in order to master vitrification using CCIM [1-6].
Among the fission products are platinoids of very limited solubilities in glass melts such as $\mathrm{Ru}, \mathrm{Rh}$, and Pd, notwithstanding they significantly affect the viscosity and electrical properties of the melt during vitrification processes. The fission product ruthenium is a particular component of spent nuclear fuel due to its complex chemistry. However, under the standard melt conditions, it is mostly present in the tetravalent oxidation state. At $1473 \mathrm{~K}$, its solubility in sodium borosilicate glass is in the order of hundreds of ppm $[7,8]$. Therefore, most of the ruthenium in glass can be found in the form of $\mathrm{RuO}_{2}$ precipitates $[3,4,7,9,10]$. The $\mathrm{RuO}_{2}$ rutile phase is about three times denser than commonly employed sodium borosilicate glasses. Nevertheless, the most striking contrast of these phases is related to their electrical properties. While sodium borosilicate glasses are essentially ionic conductors with electrical conductivity being strongly dependent on temperature $\left(10^{-7}-1 \Omega^{-1} \mathrm{~cm}^{-1}, 500-1500 \mathrm{~K}\right)$ [11], $\mathrm{RuO}_{2}$ has a metal-like conductivity slightly dependent on temperature $\left(2.5 \times 10^{4} \Omega^{-1} \mathrm{~cm}^{-1}\right.$ at room temperature) [3]. Therefore, even small amounts of this fission product can increase the overall electrical conductivity of the glass melt by several orders of magnitude if the conductive phase percolates $[3-5,12]$.

Due to the uniqueness mentioned above, the electrical conductivity of $\mathrm{RuO}_{2}$-glass composites has been extensively studied in the past 20 years, using impedance spectroscopy both in the solid $\left(\mathrm{T}<\mathrm{T}_{\mathrm{g}}\right)$ and 
molten states $\left(T>T_{g}\right)$, where $T_{g}$ is the glass transition temperature [13]. All results pointed out for an abrupt increase in the electrical conductivity when the $\mathrm{RuO}_{2}$ content reached around $1 \%$ vol. $[4,5,11,12]$. However, the theoretical amount necessary for a geometrical percolation to occur, considering randomly distributed spheres of the same size, is around $15 \%$ vol. Even for segregate systems, when the conductive spheres are ten times smaller than the isolating ones, the percolation threshold can be only as low as 4\% vol. [14]. This discrepancy between the experimental and theoretical percolation thresholds might be partially explained by a regrouping of $\mathrm{RuO}_{2}$ particles in the melt during the synthesis step $[4,13]$. Another likely scenario is that some ruthenium dissolved or precipitated as nanocrystals in the glass matrix would play at least locally, a role in the conduction process [15-17]. Nonetheless, the parcel of these contributions has not been unveiled up to now $[4,12]$. Useful information could come from an investigation of complementary electrical properties like permittivity and loss. However, for $\mathrm{RuO}_{2}$ content above the percolation threshold, this information is often inaccessible from impedance spectroscopy experiments because the composites are constituted of highly conductive pathways which overshadow capacitive effects $[4,5,11,12]$. Moreover, whenever accessible, i.e., for low conducting materials, an "average" information is obtained since the experiment is a macroscopic one.

Techniques derived from Scanning Probe Microscopies (SPM) and particularly Atomic Force Microscopy (AFM) techniques are likely to provide local information on the dielectric properties of $\mathrm{RuO}_{2}$-glass composites at scales that would allow to discriminate between the regions rich in $\mathrm{RuO}_{2}$ agglomerates and region of apparently $\mathrm{RuO}_{2}$-free borosilicate matrix. In Atomic Force Microscopy (AFM), a physical probe with a tip of nanometric apex at its end is used, thus providing the nanometric resolution. The microscope probes the material with a large precision by sensing the field of interaction between the sample and the probe. Electrostatic Force Microscopy (EFM) is an AFM-derived method where interacting electrostatic forces are selectively detected. EFM is a promising technique for the local characterization of heterogeneous materials, owing to its sensitivity to the capacitive response in the presence of the sample [18]. However, due to the probe geometry and the long-range nature of electrostatic forces, EFM signals can be easily misinterpreted. No analytical model has been developed up to now to help to interpret the EFM results, i.e. to describe the interaction between an EFM tip and a material, to the best of our knowledge. However, while not all numerical methods can be adapted [19], the finite-element one is an appropriate numerical solution to simulate EFM signals accounting for the exact geometry of the tip and the sample [20,21].

Recently, Riedel et al. [20] proposed a method to measure the local relative permittivity of isolating films using electrostatic force microscopy (EFM). They developed an experimental protocol for this specific case, which is based on the detection of the electric force gradient employing a double pass method [22]. The dielectric permittivity was then obtained by fitting experimental data with an expression of the force gradient calculated by the equivalent charge method (ECM) [20]. Based on these ideas, we have adapted the aforementioned method to study the local relative permittivity of borosilicate glasses containing $\mathrm{RuO}_{2}$. Impedance spectroscopy was also used to determine the overall electrical properties of these $\mathrm{RuO}_{2}$ glass composites at a macroscopic scale, while scanning electron microscopy was employed to investigate the morphology and the arrangement of $\mathrm{RuO}_{2}$ particles in the glass matrix at a local scale.

\section{Experimental method}

\subsection{Glass synthesis}

We have chosen simplified compositions that simulate complex nuclear waste glasses containing noble metals. Since $\mathrm{RuO}_{2}$ is the main re- sponsible for the outstanding electrical behavior of these oxide melts, the only platinoid considered here was $\mathrm{RuO}_{2}$. Therefore, the simplified glass composition studied in this work is based on a sodium-calcium borosilicate glass containing $\mathrm{RuO}_{2}$ particles, which one might consider as a $\mathrm{RuO}_{2}$-glass composite. Table 1 presents the nominal compositions of glass composite batches according to their oxide molar content. The $\mathrm{RuO}_{2}$-glass composites were prepared by melting appropriate amounts of $\mathrm{Na}_{2} \mathrm{CO}_{3}, \mathrm{CaCO}_{3}, \mathrm{H}_{3} \mathrm{BO}_{3}, \mathrm{SiO}_{2}$, and a powder of nanocrystalline $\mathrm{RuO}_{2}$ particles (Heraeus, Germany) with a specific area of $34 \mathrm{~m}^{2} / \mathrm{g}$. The formulated glasses were prepared in a platinum-gold crucible in batches of $100-150 \mathrm{~g}$ using a double melting protocol, carried out in a muffle furnace under air. In a first melting, the temperature was raised to $1123 \mathrm{~K}$ and kept at this temperature for $4 \mathrm{~h}$ to eliminate $\mathrm{CO}_{2}$, and then it was raised to $1473 \mathrm{~K}$ for melting and maintained at this temperature for $3 \mathrm{~h}$. The resulting melt was quenched and ground in a planetary mill then melted again during $1 \mathrm{~h}$ at $1473 \mathrm{~K}$, followed by quenching. The glass composites were then annealed around $873 \mathrm{~K}$ for $1 \mathrm{~h}$, and slowly cooled at $1 \mathrm{~K} / \mathrm{min}$ to relieve mechanical stress $[23,24]$. The volatilization of $\mathrm{RuO}_{2}$ through its decomposition into $\mathrm{RuO}_{4}(\mathrm{~g})$ and $\mathrm{RuO}_{3}(\mathrm{~g})$ at temperatures above $1423 \mathrm{~K}$ is well documented in the literature. However, the magnitude of the $\mathrm{RuO}_{2}$ weight loss in borosilicate of similar composition is smaller than $5 \%$ at $1473 \mathrm{~K}$ [9]. Therefore, the final $\mathrm{RuO}_{2}$ concentration in the glass-composite should be slightly lower than the nominal but for simplicity matter the nominal composition is considered here.

\subsection{Microstructural characterization}

The microstructure of the $\mathrm{RuO}_{2}$-glass composite was investigated by scanning electron microscopy (SEM) and energy dispersive spectroscopy (EDS). Before SEM analyses, the samples were sanded and finely polished employing $1 \mu \mathrm{m}$ diamond suspension; carbon was then sputtered onto the surface to ensure electrical ground. SEM observations were performed employing a ZEISS EVO HD15 scanning electron microscope equipped with an energy dispersive X-Ray detector (OXFORD X-max) for microanalyses.

\subsection{Electrical measurements by impedance spectroscopy}

The overall electrical properties, conductivity and permittivity, of the $\mathrm{RuO}_{2}$-borosilicate glass composite were determined via impedance spectroscopy (IS). Parallelepiped-shaped samples were cut, sanded, and finely polished employing $1 \mu \mathrm{m}$ diamond suspension; then, gold electrodes were sputtered on their parallel opposite faces to ensure electrical contact. Due to the contrasting conductivity of these glass composites, the specimens were intentionally prepared with distinct $\left(2-282 \mathrm{~m}^{-1}\right)$ shape factors (thickness/area) in order to maximize or minimize their overall impedance adjusting it to the accuracy of the equipment $[23,24]$. IS measurements were conducted using a Bio-Logic

Table 1

Nominal compositions of glass composite batches according to their oxide molar content. The volume content of $\mathrm{RuO}_{2}$ is also calculated based on its density $\left(6.97 \mathrm{~g} / \mathrm{cm}^{3}\right)[11]$ and the density of the base R0 glass sample $\left(2.52 \mathrm{~g} / \mathrm{cm}^{3}\right)$. The glass composite samples are named according to their $\mathrm{RuO}_{2}$ volume content.

\begin{tabular}{lcccccc}
\hline Sample & \%mol. & \multicolumn{1}{c}{} & & \%vol. \\
\hline & $\mathrm{Na}_{2} \mathrm{O}$ & $\mathrm{CaO}$ & $\mathrm{B}_{2} \mathrm{O}_{3}$ & $\mathrm{SiO}_{2}$ & $\mathrm{RuO}_{2}$ & $\mathrm{RuO}_{2}$ \\
\hline $\mathrm{R} 0$ & 14.06 & 8.66 & 10.77 & 66.50 & 0.00 & $\mathbf{0}$ \\
$\mathrm{R} 0.5$ & 13.97 & 8.60 & 10.70 & 66.04 & 0.69 & $\mathbf{0 . 5 5}$ \\
$\mathrm{R} 0.8$ & 13.92 & 8.57 & 10.66 & 65.83 & 1.02 & $\mathbf{0 . 8 1}$ \\
$\mathrm{R} 1.1$ & 13.86 & 8.54 & 10.62 & 65.58 & 1.40 & $\mathbf{1 . 1 0}$ \\
$\mathrm{R} 3.0$ & 13.52 & 8.33 & 10.36 & 63.95 & 3.84 & $\mathbf{3 . 0 4}$ \\
$\mathrm{R} 5.5$ & 13.08 & 8.07 & 10.03 & 61.88 & 6.95 & $\mathbf{5 . 5 5}$ \\
\hline
\end{tabular}


MTZ-35 impedance analyzer over the frequency range $1 \mathrm{MHz}-1 \mathrm{~Hz}$, with an applied root mean square AC voltage of $100 \mathrm{mV}$. Before every measurement, a calibration routine available on the software MTZ-35 was performed to minimize errors. These measurements were carried out in air, using a two-probe sample holder, at temperatures below the glass transition temperature $\mathrm{T}_{\mathrm{g}}(\sim 860 \mathrm{~K})$ over the range $400-800 \mathrm{~K}$.

\subsection{Dielectric measurements by electrostatic force microscopy (EFM)}

\subsubsection{Theoretical background: gradient detection method}

During an EFM measurement, an electric field between the AFM tip of the cantilever and the sample is induced by applying a voltage. When the AFM probe is placed in the vicinity of the sample surface, or when the tip is conditioned in a way that a force field is created due to tip-sample interaction, the frequency of the cantilever oscillation depends on the tip-sample interaction, which in turn depends on the electrical state of the sample surface.

The general problem to be solved when describing the resonance modes corresponds to that of a harmonic oscillator immersed in a force field, then an additional electrostatic force $F_{\text {elec }}$ must be considered. If we do not know the dependency of $F_{\text {elec }}$ with the distance between the tip and the equilibrium position $z$, the equation of movement cannot a priori be solved. However, for small oscillations, or small displacements around average $z$ scanning position, $z_{0}$, it can be expressed as a first-order Taylor development around $z_{0}[25]$ :

$F_{\text {elec }}(z) \approx F_{\text {elec }}\left(z_{0}\right)+z\left(\frac{\partial F_{\text {elec }}}{\partial z}\right)_{z_{0}}$

the equation of movement becomes:

$$
\begin{aligned}
m_{e} \ddot{z}= & -\frac{m_{e} \omega_{0}}{Q} z-\left(k-\left(\frac{\partial F_{\text {elec }}}{\partial z}\right)_{z_{0}}\right) z+F_{\text {elec }}\left(z_{0}\right) \\
& +F_{d} \cos \left(\omega_{c} t\right)
\end{aligned}
$$

where $\omega_{0}$ is the resonance frequency of the oscillator, $F_{d}$ represents the amplitude of the excitation at a frequency $\omega_{c}$ and $m_{e}$ is the effective mass of the cantilever. The term $F_{\text {elec }}\left(z_{0}\right)$ is independent of time, which only creates a static deflection of the cantilever that has no influence on the coming process. However, from the second terms at the right-hand side of Eq. (2), it can be noticed that when subjected to an interaction force with the sample, force gradients modify the effective stiffness of the probe, $k_{e f f}$, which is written:

$k_{\text {eff }}=k-\left(\frac{\partial F_{\text {elec }}}{\partial z}\right)_{z_{0}}$

the new cantilever pulsation is then given by:

$\omega_{\text {eff }}=\left(\frac{\left(k-\frac{\partial F_{\text {elec }}}{\partial z}\right)}{m_{e}}\right)^{1 / 2}=\omega_{0}\left(1-\frac{1}{k} \frac{\partial F_{\text {elec }}}{\partial z}\right)^{1 / 2}$

for small displacements, the average force gradient is small compared to the spring constant of the cantilever: $\left(\frac{\partial F_{\text {elec }}}{\partial z}\right)_{z_{0}} \ll k$. Consequently, the Taylor series of Eq. (4) indicates that the frequency shift $\Delta f_{0}$ of the probe resonance frequency for a weakly perturbed oscillator is proportional to the force gradient of the interaction:

$\Delta \omega_{0}=\omega_{\text {eff }}-\omega_{0} \cong-\frac{1}{2} \frac{\omega_{0}}{k} \frac{\partial F_{\text {elec }}}{\partial z}$

$\Delta f_{0} \cong-\frac{f_{0}}{2 k} \frac{\partial F_{\text {elec }}}{\partial z}$ furthermore, for static force gradients, the mechanical phase shift can be expressed as $[22,26]$ :

$\Delta \phi_{C} \cong-\frac{Q}{k} \frac{\partial F_{\text {elec }}}{\partial z}$

thus, from Eqs. (6) and (7) it can be deduced that force gradients can be detected by either measuring the frequency shifts $\Delta f_{0}$ or the phase shifts $\Delta \phi_{C}$, respectively. Even so, measuring the frequency shifts is preferred to avoid phase saturation observed at high voltages for example $(>10 \mathrm{~V})[20]$.

Let us consider the case of a dielectric sample on a conductive substrate. When a voltage $\mathrm{V}$ is applied to the probe (with the sample holder grounded), the electrostatic force gradient $\frac{\partial F_{\text {elec }}}{\partial z}$ involved in EFM signals can be written as:

$\frac{\partial F_{\text {elec }}}{\partial z}=\frac{1}{2} \frac{\partial^{2} C}{\partial z^{2}} V^{2}$

where $\mathrm{C}$ is the tip-surface capacitance.

Let us now consider experiments where only DC voltages are applied. As expected from Eq. (6), the curves $\Delta f_{0}(V)$ have the parabolic shape $-a_{\Delta f_{0}}(z) V^{2}$ where $a_{\Delta f_{0}}(z)$ is related to the tip-sample capacitance and force gradient by the expression [20]:

$a_{\Delta f_{0}}(z) \frac{f_{0}}{4 k} \frac{\partial^{2} C}{\partial z^{2}}=\frac{f_{0}}{2 k V^{2}} \frac{\partial F_{\text {elec }}}{\partial z}(9)$.

Fig. 1 shows typical frequeicy shifts $\Delta f_{0}$ curves obtained for the borosilicate matrix (R0) at different tip-surface distances.

The EFM measurements were performed using a Bruker Nanoscope Dimension 3100 microscope. The EFM was carried out using the conventional frequency modulation technique at the first cantilever frequency $\left(\mathrm{f}_{0}=60.2 \mathrm{kHz}\right)$ in Lift-Mode (with tip-sample distances comprised between $\sim 15 \mathrm{~nm}$ and $\sim 110 \mathrm{~nm}$ ). The amplitude of the vibrating lever $\left(A_{\text {lift }}\right)$ was lower than the amplitude set-point $\left(A_{\text {lift }} \sim 1 / 3\right.$ ASP). Experiments were performed in ambient conditions using a commercial coated metal $\mathrm{PtIr}_{5}$ tip at different voltages, from $-4 \mathrm{~V}$ to $+12 \mathrm{~V}$. The spring constant and the quality factor of the cantilever were $2.1 \mathrm{~N} / \mathrm{m}$ and 144 , respectively. Scans were performed on $\mathrm{RuO}_{2}$-glass composites samples finely polished employing $1 \mu \mathrm{m}$ diamond suspension.

\subsubsection{Numerical modeling: finite element method}

The Comsol® Multiphysics software has been used to study the interaction between an AFM conductive probe and different types of dielectric samples. Fig. 2 shows a schematic representation of the interacting system and the distribution of the electrostatic potential in the vicinity of the probe. The interacting system is mainly composed of the force detector represented by the EFM probe and the sample, placed on a conductive planar counter-electrode. We modeled the tip according to

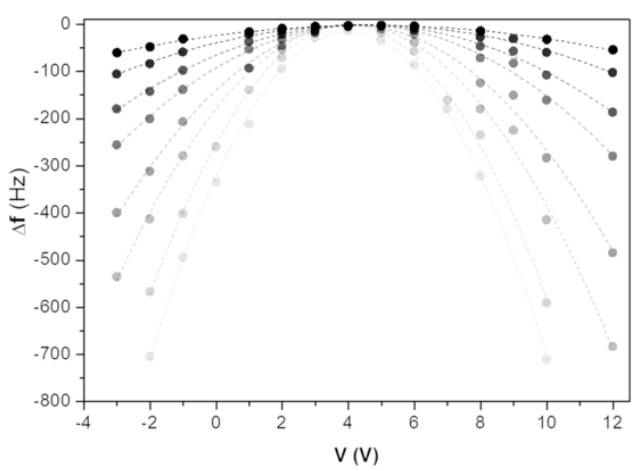

Fig. 1. Example of $\Delta f_{0}(V)$ curve obtained for the borosilicate matrix (R0) at different tip-surface distances. The variation of the gray tonality indicates an increase of the distance between the tip and the surface, from light gray to dark gray for small to large tip-surface distances, respectively. 

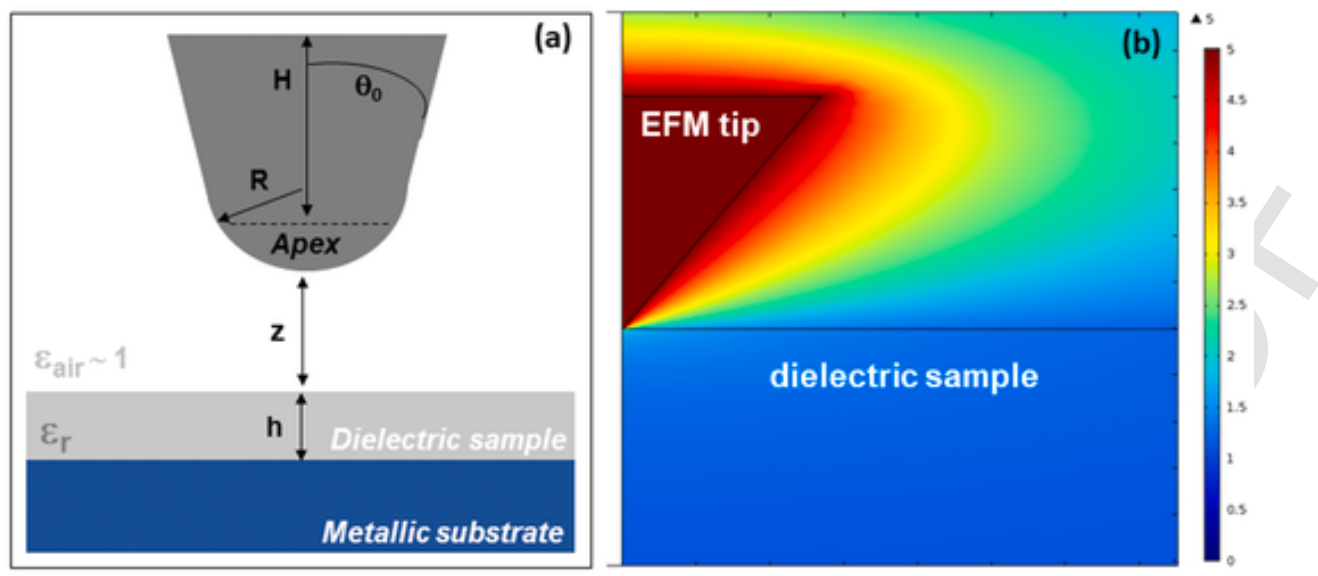

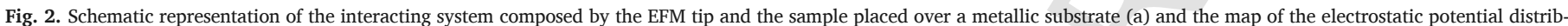
ution (b).

the geometry of standard EFM tips, namely, as a solid truncated cone of height $\mathrm{H}=10 \mu \mathrm{m}$ and half-angle $\theta=10^{\circ}$ with a spherical apex at its end [27]. We placed the tip at a commonly used EFM working distance $z$ varying from 10 to $110 \mathrm{~nm}$ from the top of the sample placed on a metallic substrate (Fig. 2.a). Note that our experimental EFM measurements were performed in the force gradient detection mode, which further localizes the interaction to the nanometric parts of the probe $[28,29]$. The substrate was modeled as a disk of $20 \mu \mathrm{m}$ diameter, which allowed taking into account finite-size lateral effects. We modeled the air in the EFM enclosure as an empty space of relative permittivity $\varepsilon_{\mathrm{r}}=1$. For $2 \mathrm{D}$ axisymmetric calculations, spherical geometry coordinates and a spherical shape for the simulation box were chosen. The box was divided into two spherical regions to model the infinite extension of the space surrounding the probe-sample system: the inner region of $30 \mu \mathrm{m}$ radius and the outer one of $32 \mu \mathrm{m}$ radius. We applied the built-in infinite element transformations to the outer region to avoid box geometry and dimension influence. An automated extremely fine physics-controlled meshing was used to the whole simulation box. Meshing domains have the form of free triangles in 2D, with a minimum size of $1.28 \mathrm{~nm}$ and a maximum size of $640 \mathrm{~nm}$. We verified that, within these conditions of simulation box extension and resulting mesh size and form, our results were sufficiently correct $(<5 \%$ variation with larger boxes and/or smaller mesh size). For 2D calculations, keeping the same model dimensions, square boxes were defined for computation region, and cylindrical coordinates were used. Infinite element transformations were not applied for 2D simulations. However, the negligible influence of border effects was also verified ( $<10 \%$ variation with larger boxes).

The problem consisted of determining the electrostatic interaction between the EFM sensor and the metallic substrate, with or without a specific dielectric sample. During conventional EFM measurements, electrostatic force detection and/or force gradient detection can be analyzed. In this paper, we calculated the electrostatic force as a basic theoretical study of EFM signals. Moreover, the used software provided direct force measurements, whereas an additional procedure, detailed in the data analysis section, was adopted to study the force gradient. Such a model was first introduced by Arinero et al. [30] where the possible presence of buried nanoparticles was also considered.

2.4.2.1. Domains equations The finite-element method based software solves the Poisson's equation for the tip-sample system, maps the electrostatic potential $V(r, z)$ distribution (Fig. 2.b), and then calculates the Maxwell stress tensor. The integration of the Maxwell stress tensor around the tip surface gives the resulting electrostatic force at each position of the scan-line. To shift from 2D calculations to 3D ones, the system includes the perimeter $2 \pi r$ factor in Eq. (3) where $r$ is the distance from the 2D probe surface to the axis of symmetry.

2.4.2.2. Boundary equations The continuity conditions at the sample material interfaces (material 1: $\overrightarrow{D_{1}}(r, \mathrm{z})$ and material $2: \overrightarrow{D_{2}}(r, \mathrm{z})$ ) and interior simulation box boundaries can be expressed by:

$\vec{n} \cdot \overrightarrow{D_{1}}(r, \mathrm{z})=\vec{n} \cdot \overrightarrow{D_{2}}(r, \mathrm{z})$

where $\overrightarrow{D_{i}}(r, \mathrm{z})$ (i=1 or 2), is the dielectric displacement vector.System external boundaries, applied on external box edges, have been set to insulators by:

$\vec{n} \cdot \vec{D}(r, \mathrm{z})=0$

Note that these conditions are ensured by the natural boundary conditions of the program. When measuring the force at the top of the sample, our system is axisymmetric. We calculated the system in 2D axisymmetric dimensions, which is faster and more accurate than $3 \mathrm{D}$ calculations performed within a reasonable time of computation. The probe was biased, whereas the substrate was grounded. We fixed the potential to $5 \mathrm{~V}$ and $0 \mathrm{~V}$ respectively on the tip and the substrate boundaries for $2 \mathrm{D}$ axisymmetric calculations and their surface for $2 \mathrm{D}$ ones.

2.4.2.3. Data analysis protocol A force gradient function versus $z$ was evaluated from the derivative of the force (power-law: $F_{\text {elec }}(z)=A \cdot z^{B}$ with A and B constants) after each simulation, as illustrated in Fig. 3.

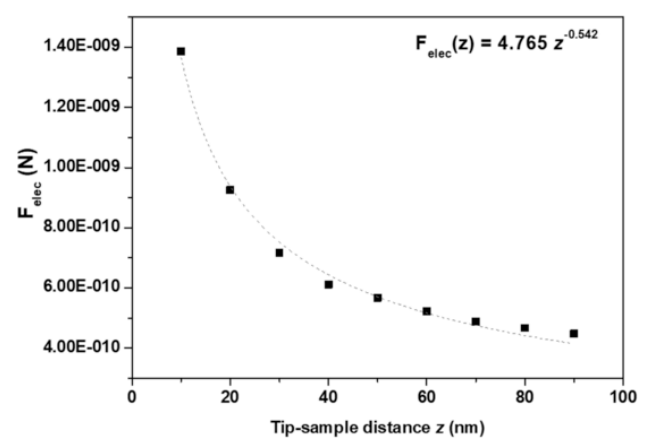

Fig. 3. Power-law interpolation function to estimate $F_{\text {elec }}(z)$. The force gradient $\frac{\partial F_{\text {elec }}(z)}{\partial z}$ is obtained by derivation of this function. Example obtained on the borosilicate matrix (R0). 


\section{Results and discussion}

\section{1. $\mathrm{RuO}_{2}$-glass composite microstructure}

The microstructure of distinct $\mathrm{RuO}_{2}$-glass composites has been widely reported in the literature, especially in the nuclear waste context and thick-film resistors $[9,15]$. Since the dissolution limit of ruthenium in silicate glass is generally marginal, the overall microstructure does not vary considerably once the composite will be constituted of $\mathrm{RuO}_{2}$ precipitates embedded in a glass matrix. However, the distribution and shape of the $\mathrm{RuO}_{2}$ precipitates, their interaction with the glass matrix, and the partial dissolution of ruthenium in the glass matrix could differ substantially depending on the glass composition, the nature of ruthenium precursor, and temperature of synthesis [9,12]. Fig. 4 depicts micrographs of $\mathrm{RuO}_{2}$-glass composites with different $\mathrm{RuO}_{2}$ content employing scanning electron microscopy (SEM) followed by energy dispersive spectroscopy (EDS) analyses. Since samples R5.5 (Fig. 4. e) have been tailored with about ten times more $\mathrm{RuO}_{2}$ than samples $\mathrm{R} 0.5$ (Fig. 4. a), the difference in the amount of $\mathrm{RuO}_{2}$ particles is evident when these micrographs are compared under low magnification. Nevertheless, the distribution of particles along the glass matrix in the form of clusters is quite similar in both cases (Fig. 4. b and f). Some of these particles seem to be in direct contact with one another, which could give rise to short range conduction chains made of $\mathrm{RuO}_{2}$ particles. Other $\mathrm{RuO}_{2}$ particles appear to have grown considerably in the glass synthesis step. The sizes of these bigger particles could indicate coalescence occurrence that would also require direct contact between the particles. Besides, EDS mapping analyses reveal that the dispersion of ruthenium in the glass composite is restricted to $\mathrm{RuO}_{2}$ undis- solved particles in the clusters, at least within the detection limit of the technique that is about a thousand ppm (Fig. $4 \mathrm{c}$ and g). However, any ruthenium, either dissolved in the glass matrix or in the form of nanometric precipitates present in quantity lower than the detection limit of the technique, would be overlooked. Obviously, this cannot be ruled out, owing to the low solubility of $\mathrm{RuO}_{2}$ in the borosilicate glass $[16,17]$. Conversely, silicon (Fig. $4 \mathrm{~d}$ and h) seems to be well dispersed in the glass matrix as in a homogeneous glass.

\subsection{Overall electrical properties by impedance spectroscopy}

Fig. 5 displays the dependence of the electrical conductivity on the $\mathrm{RuO}_{2}$ content and temperature. Electrical conductivity data are obtained from impedance spectroscopy analysis according to a classical methodology well described elsewhere [23]. In summary, the resistances (R) of the samples are taken from the intercept of the impedance response with the real axes $\left(\mathrm{Z}^{\prime}\right)$ at low frequency and the DC conductivity is calculated according to the relationship $\sigma_{\mathrm{DC}}=1 / \mathrm{RA}$, where 1 is the thickness and $\mathrm{A}$ is the area of the sample. An abrupt increase (percolation threshold) of the electrical conductivity DC is observed around $\mathrm{RuO}_{2}$ contents of about $1 \%$ (Fig. 5.a), confirming the tendencies already found in earlier studies $[4,5,12,23]$. The distinct impedance behavior of $\mathrm{RuO}_{2}$-glass composites samples below and above the percolation threshold is discussed in detail in our previous work [23]. The dependence of the electrical conductivity on temperature (Fig. 5.b) for samples with $\mathrm{RuO}_{2}$ contents below the percolation threshold (R0 and R0.5) is typical of a thermally activated conduction mechanism such as the ionic migration in glasses, in this case, mostly $\mathrm{Na}^{+}$. On the other hand, samples with $\mathrm{RuO}_{2}$ content above the percolation threshold

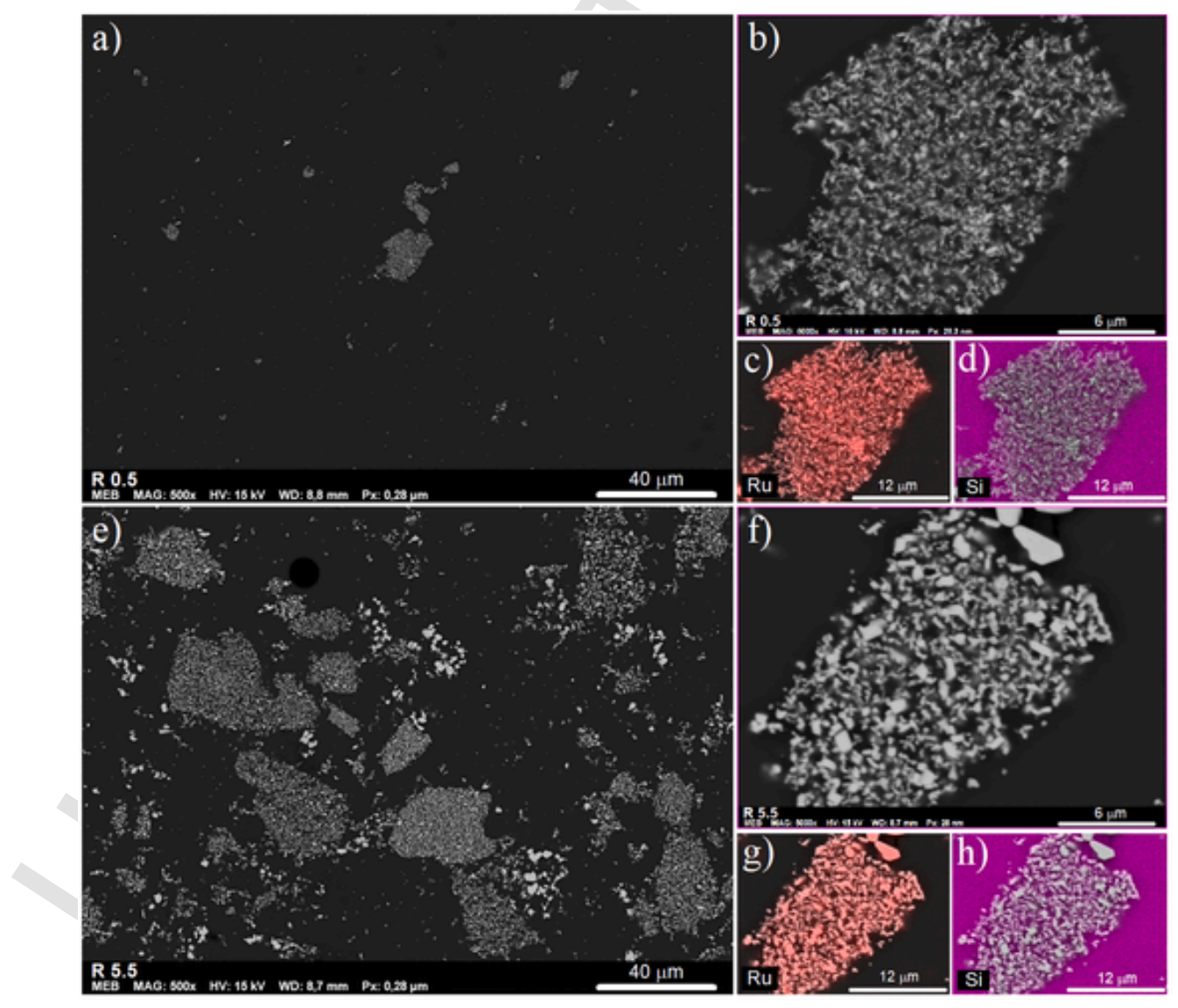

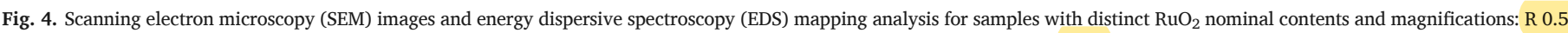

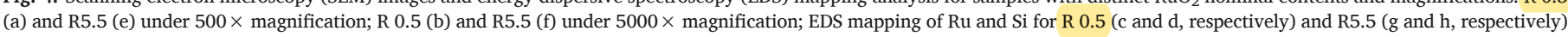
samples. 
a)

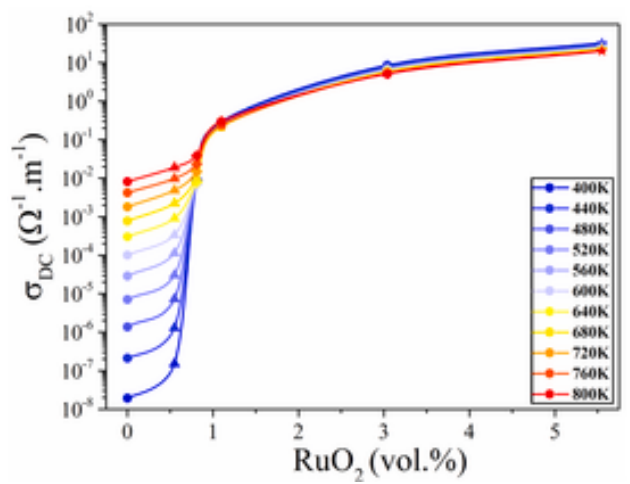

b)

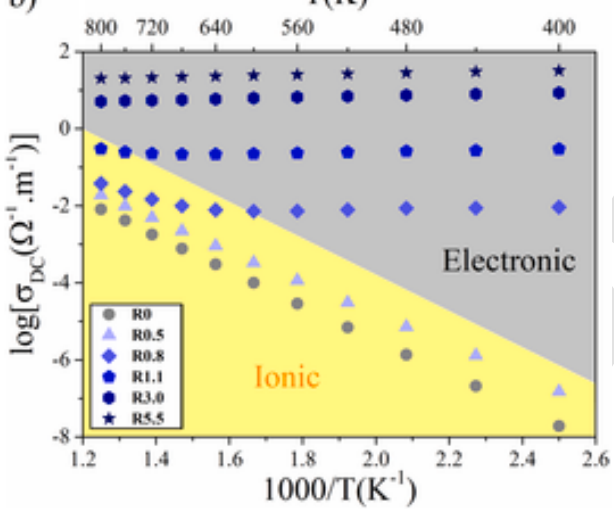

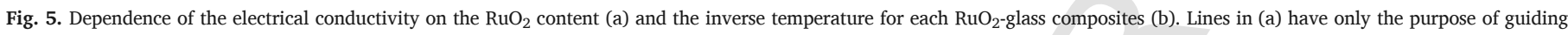

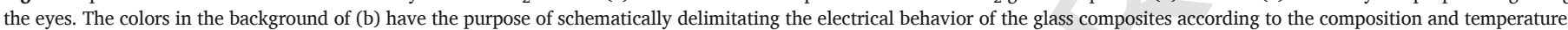

(R1.1, R3.0, and R5.5) demonstrate a characteristic metal-like conductivity that slightly decreases with temperature (Fig. 5.b). Intermediate to those behaviors is the sample R0.8 (Fig. 5.b), which exhibits a metal-like conductivity at low temperatures $(\mathrm{T}<600 \mathrm{~K})$ and a prevailing ion-conducting behavior at high temperatures ( $T>600 \mathrm{~K}$ ). This feature arises due to the divergent temperature-dependence of the ionic and metal-like conductivities. Given its electrical behavior, it is safe to assume that this sample (R0.8) has a $\mathrm{RuO}_{2}$ content in the vicinity of the percolation threshold. As previously mentioned, this percolation threshold is exceptionally low to be rationalized in the framework of the percolation theory $[14,15]$.

From complex impedance data, we can also calculate the complex relative permittivity $\left(\varepsilon_{\mathrm{r}}{ }^{*}(\mathrm{f})\right)$ of the $\mathrm{RuO}_{2}$-glass composite. The dependence of the real part of the relative permittivity $\left(\varepsilon_{\mathrm{r}}\right)$ on frequency $(\mathrm{f})$ can be directly accessed by converting complex impedance data accord- ing to Eq. (12), where 1/A is the shape factor of the sample (thickness/ area); $\varepsilon_{0}$ is the vacuum permittivity; Z' and Z" are the real and the imaginary part of the complex impedance, respectively [31].

$\varepsilon_{r}^{\prime} \quad(\mathrm{f})=\frac{l}{A} \frac{-Z^{\prime \prime}(\mathrm{f})}{f \varepsilon_{0}\left(Z^{\prime}(\mathrm{f})^{2}+Z^{\prime \prime}(\mathrm{f})^{2}\right)}$

Fig. 6 exhibits the dependence of the real part of the relative permittivity $\left(\varepsilon_{\mathrm{r}}\right.$ ) on frequency at different temperatures for samples R0 (Fig. 6. a), R0.5 (Fig. 6. b), and R0.8 (Fig. 6. c), as well as its overall relative permittivity (Fig. 6. d) or permittivity DC $\left(\varepsilon_{\mathrm{r}}^{\prime} \mathrm{DC}\right)$. The latter is given by the total relative permittivity of the sample at $\mathrm{f}=0 \mathrm{~Hz}$ excluding the electrode polarization. Samples with high $\mathrm{RuO}_{2}$ content (R1.1, R3.0, and R5.5) and consequently high conductivity are not shown here because the real part of permittivity in these cases is overshad-
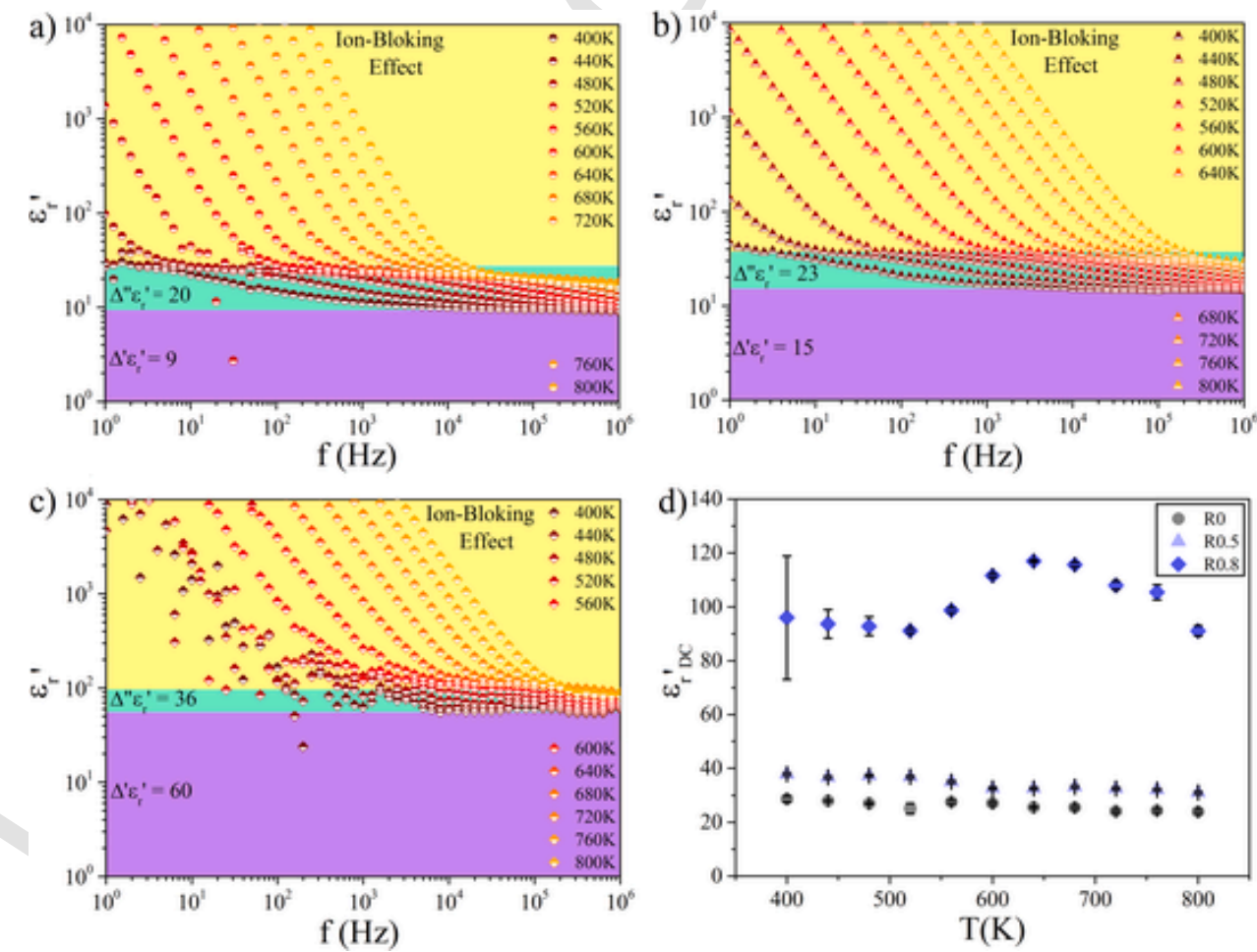

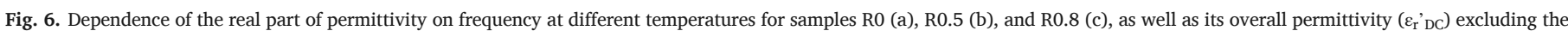

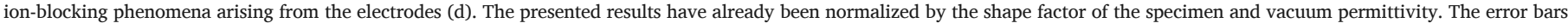
in (d) arise from the average of three data points taken in the middle of the second plateau. 
owed by the conductivity of the sample and masked by the inductance of the sample holder and cables [12,23,31]. Nonetheless, it is possible to observe some tendencies in the samples in which the permittivity behavior can be measured. At low temperatures, two plateaus are seen before a rapid increase in permittivity at low frequencies. The vast contribution to $\varepsilon_{\mathrm{r}}^{\prime}$ at low frequency arises from the polarization phenomenon of electrodes caused by an ion-blocking effect. This polarization phenomenon should not be considered as part of the permittivity of the samples $[6,31]$. In the case of sample R0.8 (Fig. 6. b), a dispersion of the data is observed in this frequency region at low temperatures due to an electronic leakage across the metallic electrodes that occurs when the metal-like contribution to the electrical conductivity overcomes the ionic contribution (refer to Fig. 5. b) [23]. The contribution to $\varepsilon_{\mathrm{r}}$ ' at high frequency $\left(\Delta^{\prime} \varepsilon_{\mathrm{r}}{ }^{\prime}\right)$ given by the first plateau is related to fast and localized polarization phenomena such as electronic, atomic, and dipolar phenomena. The second contribution located at the middle-frequency range $\left(\Delta{ }^{\prime} \varepsilon_{r}\right)$ is more likely to be related to long-range ionic displacement $[23,31]$. The overall relative permittivity is the sum of the two contributions and can be directly accessed at the second plateau. In general, the overall relative permittivity tends to be mostly temperature-independent, especially when compared to the electrical conductivity, which is strongly influenced by temperature in ionic conductors [31].

Nevertheless, the most important result presented here is the increase of the overall relative permittivity $\varepsilon_{\mathrm{r}}{ }^{\prime} \mathrm{DC}$ as the $\mathrm{RuO}_{2}$ content increases (Fig. 6. d). This increase of permittivity could be related to some additional spatial polarization mechanisms induced by the co-existence of conducting $\mathrm{RuO}_{2}$ particles and the insulating glass matrix. On the other hand, it could also arise from increasing amounts of dissolved $\mathrm{RuO}_{2}$ or nanometric $\mathrm{RuO}_{2}$ precipitates scattered in the glass matrix that would create additional electronic polarization. The macroscopic nature of the experiment and the average nature of the obtained permittivity values preclude the possibility of discriminating among these two hypotheses.

\subsection{Local dielectric properties by EFM analyses}

The EFM measurements were performed on this series of $\mathrm{RuO}_{2}$-borosilicate glasses (R0, R0.5, R0.8, R1.1, R3.0, and R5.5). To validate the EFM technique, and in particular, its sensitivity, two experiments were carried out at two different places of the same sample (R1.1). The first place was a zone of the glass matrix apparently free of $\mathrm{RuO}_{2}$-particles (area marked in red in Fig. 7), and the second corresponds to a place that contains $\mathrm{RuO}_{2}$-particles (area marked in green in Fig. 7). $\Delta f_{0}(V)$ curves were recorded at several tip-sample distances. Parabolas were obtained with the parabola coefficient, i.e. the concavity $a \Delta f_{0}$, depending upon the tip-sample distance $z$ as shown in Fig. 7. According to the literature [20], a steeper increase of the curve $a \Delta f_{0}(z)$ when the tip-sample distance $z$ decreases is the signature of a higher permittivity. As expected, a lower relative permittivity in the zone apparently free of $\mathrm{RuO}_{2}$-particles compared to that containing $\mathrm{RuO}_{2}$-particles is observed.

Aiming to elucidate the low percolation threshold observed in the electrical conductivity of $\mathrm{RuO}_{2}$-glass composite (see Fig. 5), a second series of EFM measurements was carried out on the "apparently" $\mathrm{RuO}_{2}$ free zones of the $\mathrm{RuO}_{2}$-glass composites. Indeed, at the percolation threshold, i.e. at about $0.8-1.0 \mathrm{vol} \% \mathrm{RuO}_{2}$, a physical pathway of $\mathrm{RuO}_{2}$ crystallite connect one to another throughout the whole composite is unlikely to occur. Therefore, regions in between crystallites might participate in the conductivity process. The goal with the EFM experiments was to estimate the local relative permittivity in these regions and by this way, to identify if the borosilicate matrix played, at least locally, a role in the conduction process.

Following the same protocol for the EFM measurements as in the previous experiments, we obtained the parabola coefficient $a \Delta f_{0}$ as a

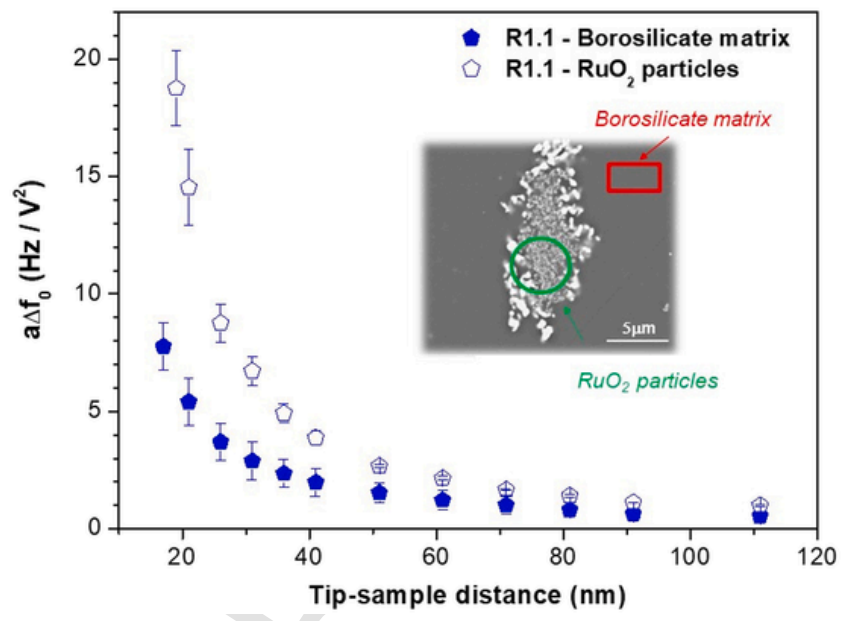

Fig. 7. Parabola coefficient $a \Delta f_{0}$ as a function of the tip-sample distance for the "apparently" $\mathrm{RuO}_{2}$-free zones of $\mathrm{RuO}_{2}$-glass composites (filled symbols) and $\mathrm{RuO}_{2}$ particles (open symbols) for sample R1.1. (For interpretation of the references to colour in this figure legend, the reader is referred to the web version of this article.).

function of the tip-sample distance for "apparently" $\mathrm{RuO}_{2}$-free zones in all studied $\mathrm{RuO}_{2}$-glass composites (Fig. 8). When the $\mathrm{RuO}_{2}$ content in the $\mathrm{RuO}_{2}$-glass composite increases, a steeper and steeper increase of the curve $a \Delta f_{0}(z)$ when the tip-sample distance decreased is observed. These results indicate a continuous increase of the local relative permittivity of "apparently" $\mathrm{RuO}_{2}$-free zones with the overall $\mathrm{RuO}_{2}$ content present in the composite. Therefore, a continuous enrichment of $\mathrm{RuO}_{2}$ in these borosilicate glass matrix zones should also be occurring.

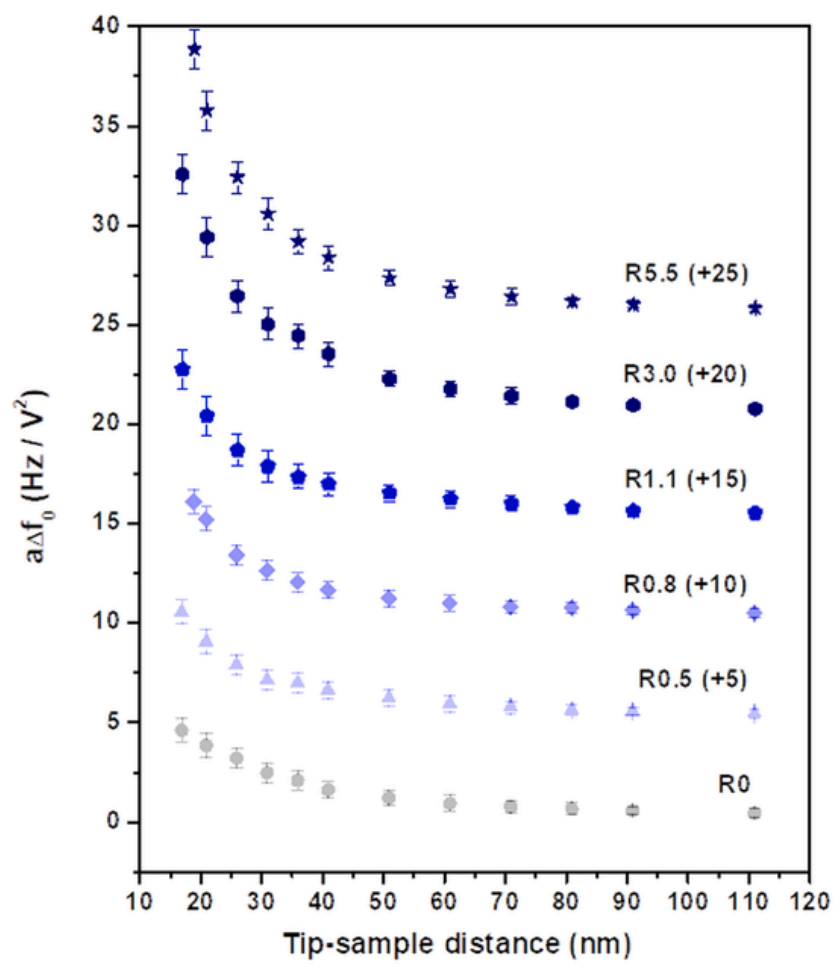

Fig. 8. Parabola coefficient $\mathrm{a} \Delta \mathrm{f}_{0}$ as a function of the tip-sample distance for "apparently" $\mathrm{RuO}_{2}$-free zones of the $\mathrm{RuO}_{2}$-glass composites (R0, R0.5, R0.8, R1.1, R3.0, and R5.5). The curves are shifted vertically for clarity. 


\subsection{Relative permittivity by finite element method}

The estimation of the relative permittivity was made thanks to the modeling of the force acting on the tip over the investigated zone. As mentioned above (Section 2.4.2), we used the AC/DC module (Electrostatics) of Comsol Multiphysics software, which uses the finite-element method to solve the Poisson's equation. The proposed protocol involved two steps. The first step consisted of determining the tip radius $\mathrm{R}_{0}$, and the second one of determining the relative dielectric permittivity of the investigated zone of the $\mathrm{RuO}_{2}$-glass composites. In all simulations, the height of the truncated solid $\mathrm{H}$ and the half-angle $\theta$ of the EFM tip (see Fig. 2.a), as well as the voltage $\mathrm{V}$ applied to the sample, were kept constants $\left(\mathrm{H}=10 \mu \mathrm{m}, \theta=10^{\circ}\right.$ and $\left.\mathrm{V}=5 \mathrm{~V}\right)$. The actual thickness of the samples comprised between 400 and $700 \mu \mathrm{m}$, was also fixed during the simulations. Then, the tip radius $R_{0}$ and the relative permittivity $\varepsilon_{r}$ are the simulation's two remaining parameters.

In a first step, we aim at extracting the actual value of the tip radius $\mathrm{R}_{0}$ (Fig. 2.a). This part of modeling was performed using the borosilicate glass that did not contain $\mathrm{RuO}_{2}$, i.e. the R0 sample. We fixed the relative permittivity at $\varepsilon_{\mathrm{r}}=29$, which was previously obtained by impedance spectroscopy (see Fig. 6. a), and we allowed the tip radius $\mathrm{R}_{0}$ to vary. The value of the tip radius $\mathrm{R}_{0}$ was obtained by comparing the experimental (gray symbols in Fig. 8) and calculated coefficients $\mathrm{a} \Delta \mathrm{f}_{0}(z)$ as a function of the tip-sample distance. Several iterations were performed until the best fit between EFM experiments and the numerical modeling based on a finite element method was obtained. Fig. 9.a) shows the experimental and optimized calculated coefficients $a \Delta f_{0}(z)$, giving a value of $R_{0}=13.5 \pm 0.5 \mathrm{~nm}$.

Once the tip radius $\mathrm{R}_{0}$ is known, the only unknown parameter is the relative permittivity $\varepsilon_{\mathrm{r}}$. As in the previous step, several iterations were performed until the best comparison between experimental and calculated coefficients $a \Delta f_{0}(z)$ as a function of the tip-sample distance was obtained, which was used to extract the value of the dielectric permittivity $\varepsilon_{\mathrm{r}}$.

Fig. 9 (a) shows the best-fit curves for the investigated zones of R0.5 (b), R0.8 (c), and R1.1 (d) samples. For tip sample-distances $z>25 \mathrm{~nm}$, EFM measurements and calculations are very close, which allowed estimating $\varepsilon_{\mathrm{r}}$ values of $33 \pm 7,45 \pm 12$ and $73 \pm 15$, respectively. For $z<25 \mathrm{~nm}$ the agreement between experimental and calculated curves is not as good, but it can be explained by the fact that the contribution of the apex of the tip in this $z$ range is preponderant, and the simple spherical form used for the modeling is too simplistic to account for the actual experiment (see Fig. 2.a).

Fig. 9.e and $f$ show the EFM experimental curves and the simulated curves for different relative permittivity values, ranging from $\varepsilon_{\mathrm{r}}=100$ to $\varepsilon_{\mathrm{r}}=2000$, for $\mathrm{RuO}_{2}$-rich glass composites ( $\mathrm{R} 3$ and R5.5 samples). None of them gave satisfying comparisons of the experimental curves, even when huge values of $\varepsilon_{\mathrm{r}}$, e.g. 2000 , were used, which indicated that the model did not apply anymore, probably because of a metallic character of these regions in these $\mathrm{RuO}_{2}$-glass composites.

\subsection{Local versus macroscopic permittivity}

Fig. 10 shows the relative permittivity $\varepsilon_{\mathrm{r}}$ of "apparently" $\mathrm{RuO}_{2}$-free zones for $\mathrm{RuO}_{2}$-glass composites (R0.5, R0.8, R1.1, R3.0 and R5.5) obtained by combination of EFM measurements and numerical modeling (blue symbols) along with the macroscopic relative permittivity obtained from impedance spectroscopy (orange symbols) (R0, R0.5, R0.8).

Since we deal with heterogeneous samples, we do not expect similar values from EIS and EFM. Indeed, EIS, being a macroscopic measurement, will give an average value that will include contributions from both the $\mathrm{RuO}_{2}$ crystallites and the borosilicate matrix. On the other hand, being a very localized measurement, EFM will discriminate between the contributions due to the crystallites on one hand and the "apparently" $\mathrm{RuO}_{2}$-free zones on the other (refer to Fig. 7).

For the R0.5 sample, the macroscopic $\varepsilon_{\mathrm{r}}$ values $(37 \pm 3)$ obtained from complex impedance, is very similar to the local $\varepsilon_{\mathrm{r}}$ values $(33 \pm 7)$ obtained from EFM measurements. It can be understood by the small amount of Ruthenium present in the sample and the weak influence of the $\mathrm{RuO}_{2}$ crystallites on the total permittivity. On the other hand, the macroscopic $\varepsilon_{\mathrm{r}}$ values $(95 \pm 22)$ obtained for the R0.8 sample is larger than the local one $(45 \pm 12)$. In this case, the number and size of the $\mathrm{RuO}_{2}$ crystallites start being important and influence the macroscopic permittivity of the sample that becomes larger than the local one. Such results validate the EFM measurements and the numerical modeling methodology to probe the local permittivity. Therefore, any change in the permittivity obtained from EFM combined with numerical modeling indeed reflects a change in the explored zone and not, an artefact due to some long range interaction.
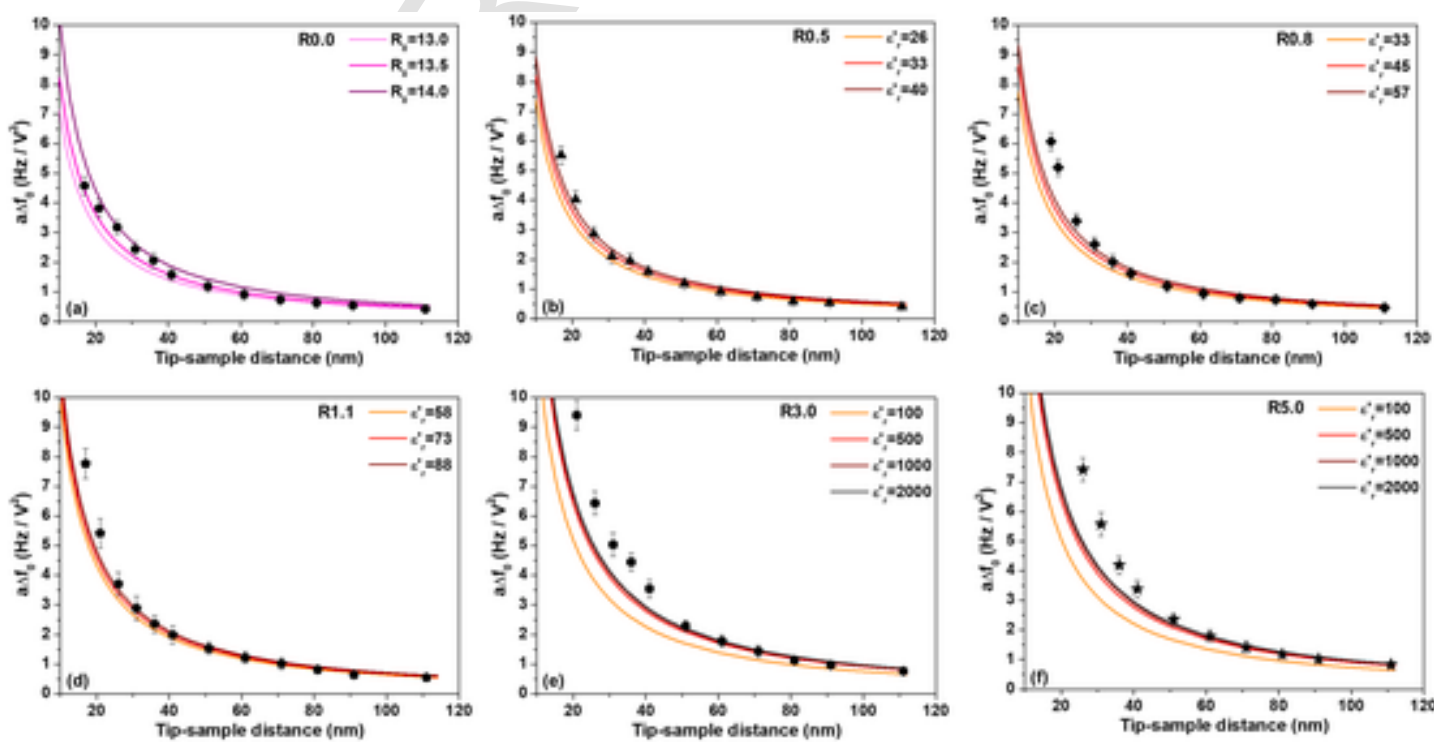

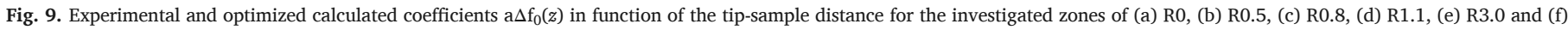
R5.5 samples. 


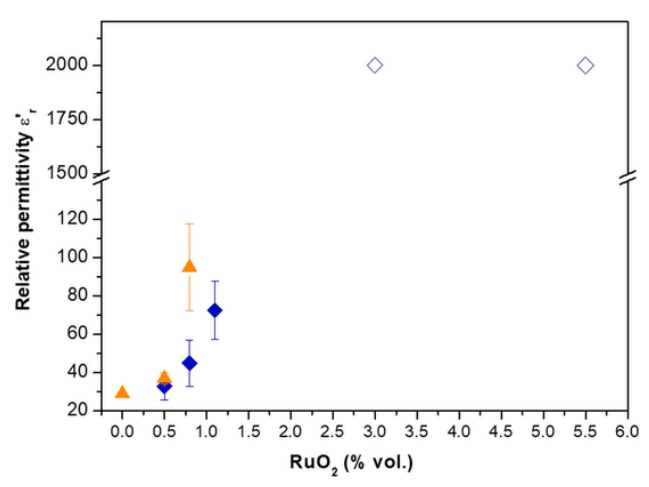

Fig. 10. Relative permittivity $\varepsilon_{\mathrm{r}}$ of "apparently" $\mathrm{RuO}_{2}$-free zones for $\mathrm{RuO}_{2}$-glass composites (R0.5, R0.8, R1.1, R3.0 and R5.5) obtained by combination of EFM measurements and numerical modeling (blue symbols) and macroscopic relative permittivity employing impedance spectroscopy (orange symbols) (R0, R0.5, R0.8). Full blue symbols correspond to $\varepsilon_{\mathrm{r}}$ values obtained from well-adjusted fits and open blue symbols correspond to $\varepsilon_{\mathrm{r}}$ values obtained from poor fits. (For interpretation of the references to colour in this figure legend, the reader is referred to the web version of this article.).

According to data reported in Fig. 10, the relative permittivity is almost constant for sample R0 and R0.5 $\left(\varepsilon_{\mathrm{r}}=29\right.$ for R0, imposed value from impedance spectroscopy data, and $\varepsilon_{\mathrm{r}}=33 \pm 7$ for R0.5), followed by a continuous increase in $\varepsilon_{\mathrm{r}}$ for R0.8 and R1.1 samples. For the $\mathrm{RuO}_{2}$-rich samples, a sudden change in the dielectric nature of the matrix occurred. Indeed, the model did not apply anymore, and it was not possible to fit the experimental curves properly even when immense values of $\varepsilon_{\mathrm{r}}$ were used.

Given the localized nature of the EFM measurements, which is approximately $100 \mathrm{~nm}$ in depth and $200 \mathrm{~nm}$ laterally, the enhancement of dielectric constant with the increase of the overall $\mathrm{RuO}_{2}$ content has to be related to the dissolution of some ruthenium in the glass matrix or nano precipitates scattered all over the matrix. It is known that ruthenium dissolves marginally in borosilicate glasses (about $100 \mathrm{ppm}$ for borosilicate glasses with similar composition) [8], but Ru-containing precipitates of extremely low size (few nanometers) have also been found in $\mathrm{RuO}_{2}$-borosilicate glasses $[16,17]$. Either way, the increase of the relative permittivity of the "apparently" $\mathrm{RuO}_{2}$-free zones indicates a local enrichment of the borosilicate matrix in $\mathrm{RuO}_{2}$. Similarly, as it influences the dielectric properties, this continuous compositional change of the matrix could also play a role in the conduction mechanism, decreasing the minimal volume of $\mathrm{RuO}_{2}$ phase necessary for the electrical percolation thanks to a larger effective volume of the conductive medium.

\section{Conclusion}

Sodium borosilicate glass composites with different $\mathrm{RuO}_{2}$ content were synthesized, and their microstructure was characterized by scanning electron microscopy (SEM) followed by energy dispersive spectroscopy (EDS). The electrical properties of the $\mathrm{RuO}_{2}$-glass composites were investigated by impedance spectroscopy (IS) and electrostatic force microscopy (EFM). IS measurements were employed to determine the overall electrical properties of the $\mathrm{RuO}_{2}$-glass composites, while EFM analyses were applied for determining their local dielectric constant. The typical microstructure of the composites showed undissolved $\mathrm{RuO}_{2}$ particles embedded in the borosilicate glass matrix. The electrical conductivity displayed an abrupt change of up to ten orders of magnitude when the volumetric $\mathrm{RuO}_{2}$ content reached about $1 \%$, highlighting an extremely low percolation threshold. Below this $\mathrm{RuO}_{2}$ content, the composites were essentially ionic conductors, and they became predominantly metal-like conductors beyond the percolation threshold.

Regarding the overall dielectric properties, the $\mathrm{RuO}_{2}$-glass composite showed a sensitive increase of the real part of the permittivity with the $\mathrm{RuO}_{2}$ content for the samples below the percolation threshold where the permittivity can still be accessed by IS measurements. On the other hand, the local dielectric constant could be determined by EFM analyses. Indeed, the EFM technique showed contrasting dielectric properties between the glass composites zones "apparently" free of $\mathrm{RuO}_{2}$ particles and the zones containing $\mathrm{RuO}_{2}$ particles. Moreover, the EFM analyses, coupled with the applied numerical modeling methodology, allowed to estimate the dielectric property of the zones "apparently" free of $\mathrm{RuO}_{2}$ particles. The protocol used was sensitive enough to detect an increase in the relative permittivity of the zones "apparently" free of $\mathrm{RuO}_{2}$ particles with the overall $\mathrm{RuO}_{2}$ content of the composites. These results indicate a continuous enrichment of the glass matrix in ruthenium, either in the dissolved state or in the form of nano precipitates unheeded by SEM analyses. By analogy with changes in the dielectric properties, this continuous compositional change of the borosilicate matrix could also play a role in the conduction mechanism, decreasing the minimal volume of $\mathrm{RuO}_{2}$ phase necessary for the electrical percolation thanks to a larger effective volume of the conductive medium.

\section{Declaration of Competing Interest}

The authors declare that they have no known competing financial interests or personal relationships that could have appeared to influence the work reported in this paper.

\section{Acknowledgments}

The Commissariat à l'Énergie Atomique et aux Energies Alternatives (CEA) and Orano are gratefully acknowledged for financial support.

\section{References}

[1] J.D. Vienna, Nuclear waste vitrification in the United States: recent developments and future options, Int. J. Appl. Glass Sci. 1 (3) (2010) 309-321.

[2] S. Naline, F. Gouyaud, V. Robineau, C. Girold, B. Carpentier, Vitrification 2010 - a challenging french vitrification project to retrofit a cold crucible inductive melter at the la hague plant, in: WM2010, 2010, Phoenix.

[3] C. Krause, B. Luckscheiter, Properties and behavior of the platinum group metals in the glass resulting from the vitrification of simulated nuclear fuel reprocessing waste, J. Mater. Res. 6 (12) (1991) 2535-2546.

[4] R. Pflieger, M. Malki, Y. Guari, J. Larionova, A. Grandjean, Electrical conductivity of RuO2-borosilicate glasses: effect of the synthesis route, J. Am. Ceram. Soc. 92 (7) (2009) 1560-1566.

[5] C. Simonnet, A. Grandjean, J. Phalippou, Electrical behavior of platinum-group metals in glass-forming oxide melts, J. Nucl. Mater. 336 (2005) 243-250.

[6] D. Jouglard, M. Neyret, L. Campo, M. Malki, Electrical property investigations and microstructure characterization of a nuclear borosilicate glass ceramic, J. Nucl. Mater. 510 (2018) 27-37.

[7] J. Mukerji, Behavior of ruthenium in glass, Ind. Eng. Chem. Prod. Res. Dev. 11 (2) (1972) 178-183.

[8] K. Sawada, T. Shimada, T. Tsukada, Si Komamine, Transfer rate of ruthenium from calcination layer to glass melt, Procedia Chem. 7 (2012) 599-603.

[9] R. Pflieger, L. Lefebvre, M. Malki, M. Allix, A. Grandjean, Behaviour of ruthenium dioxide particles in borosilicate glasses and melts, J. Nucl. Mater. 389 (2009) 450-457.

[10] H. Boucetta, R. Podor, L. Stievano, J. Ravaux, X. Carrier, S. Casale, S. Gosse, A. Monteiro, S. Schuller, Mechanism of RuO2 crystallization in borosilicate glass: an original in situ ESEM approach, Inorg. Chem. 51 (2012) 3478-3489.

[11] C. Simonnet, A. Grandjean, M. Malkic, Mixed ionic and electronic conductivity of oxides from molten state to glassy state: application to RuO2-glass composites, Solid State Ion. 175 (2004) 695-698.

[12] C. Simonnet, A. Grandjean, Mixed ionic and electronic conductivity of RuO2-glass composites from molten state to glassy state, J. Non-Cryst. Solids 351 (2005) 1611-1618.

[13] C. Simonnet, J. Phalippou, M. Malki, A. Grandjean, Electrical conductivity measurements of oxides from molten state to glassy state, Rev. Sci. Instrum. 74 (5) (2003) 2805-2809.

[14] A. Kusy, Classical percolation threshold and resistance versus temperature behaviour of RuOz-glass films, Phys. B 240 (1997) 226-241.

[15] O. Abe, Y. Taketa, Electrical conduction in thick film resistors, J. Phys. D: Appl. Phys. 24 (1991) 1163-1171.

[16] M. Totokawa, Microanalyses on the $\mathrm{RuO} 2$ particle-glass matrix interface in thick-film resistors with piezoresistive effects, Int. J. Appl. Ceram. Technol. 6 (2009) 195-204.

[17] K. Adachi, S. Iida, K. Hayashi, Ruthenium clusters in lead-borosilicate glass in thick film resistors, J. Mater. Res. 9 (1994) 1866-1878.

[18] P. Girard, Electrostatic force microscopy: principles and some applications to semiconductors, Nanotechnology 12 (2001) 485-490. 
[19] G.M. Sacha, E. Sahagún, J.J. Sáenz, A method for calculating capacitances and electrostatic forces in atomic force microscopy, J. Appl. Phys. 101 (24310) (2007) 1-4.

[20] C. Riedel, R. Arinero, P. Tordjeman, M. Ramonda, G. Lévêque, G.A. Schwartz, D.G. Oteyza, A. Alegria, J. Colmenero, Determination of the nanoscale dielectric constant by means of a double pass method using electrostatic force microscopy, J. Appl. Phys. 106 (24315) (2009) 1-6.

[21] D. El Khoury, V. Fedorenko, J. Castellon, M. Bechelany, J.-C. Laurentie, S. Balme, M. Fréchette, M. Ramonda, R. Arinero, Characterization of dielectric nanocomposites with electrostatic force microscopy, Scanning 2017 (2017) $1-14$.

[22] L. Portes, P. Girard, R. Arinero, M. Ramonda, Force gradient detection under vacuum on the basis of a double pass method, Rev. Sci. Instrum. 77 (96101) (2006) 1-5.

[23] R.B. Nuernberg, N.M.P. Machado, M. Malki, M. Neyret, Electrical behavior of RuO2-glass composites: the effect of $\mathrm{RuO} 2$ particle size on the percolation threshold, J. Nucl. Mater. 546 (2021) 152777.

[24] R.B. Nuernberg, N.M.P. Machado, D. Jouglard, L. del Campo, M. Malki, M. Neyret, The origin of hysteresis in the electrical behavior of RuO2-glass composite melts, J. Non-Cryst. Solids 557 (2021) 120596.

[25] R. Garciá, R. Perez, Dynamic atomic force microscopy methods, Surf. Sci. Rep. 47 (2002) 197-301.

[26] L. Portes, M. Ramonda, R. Arinero, P. Girard, New method for electrostatic force gradient microscopy observations and Kelvin measurements under vacuum, Ultramicroscopy 107 (2007) 1027-1032.

[27] L. Fumagalli, M.A. Edwards, G. Gomila, Quantitative electrostatic force microscopy with sharp silicon tips, Nanotechnology 25 (2014) 495701.

[28] C. Riedel, A. Alegria, G.A. Schwartz, J. Colmenero, J.J. Saenz, Numerical study of the lateral resolution in electrostatic force microscopy for dielectric samples, Nanotechnology 22 (2011) 285705.

[29] S. Gómez-Moñivas, L.S. Froufe, R. Carminati, J.J. Greffet, J.J. Sáenz, Tip-shape effects on electrostatic force microscopy resolution, Nanotechnology 12 (2001) 496-499.

[30] R. Arinero, C. Riedel, C. Guasch, Numerical simulations of electrostatic interactions between an atomic force microscopy tip and a dielectric sample in presence of buried nano-particles, J. Appl. Phys. 112 (2012) 114313.

[31] E. Barsoukov, J.R. Macdonald, Impedance, Spectroscopy: Theory, Experiment, and Applications, Wiley,, Hoboken, 2005. 